\title{
Charged mobile complexes in magnetic fields: A novel selection rule for magneto-optical transitions
}

\author{
A. B. Dzyubenko \\ Institut für Theoretische Physik, J.W. Goethe-Universität, 60054 Frankfurt, Germany \\ General Physics Institute, Russian Academy of Sciences, Moscow 117942, Russia
}

A. Yu. Sivachenko

The Weizmann Institute of Science, Rehovot 76100, Israel

(JETP Lett. 70, 514 (1999))

\begin{abstract}
The implications of magnetic translations for internal optical transitions of charged mobile electronhole $(e-h)$ complexes and ions in a uniform magnetic field $B$ are discussed. It is shown that transitions of such complexes are governed by a novel exact selection rule. Internal intraband transitions of two-dimensional (2D) charged excitons $X^{-}$in strong magnetic fields are considered as an illustrative example.
\end{abstract}

71.35.Ee, 78.20.Ls

Recently, there has been considerable experimental and theoretical interest in the behavior of $2 \mathrm{D}$ semiconductor complexes - negatively $X^{-}(2 e-h)$ and positively $X^{+}(e-2 h)$ charged excitons in magnetic fields (see, e.g., Refs. [1 5] and references therein). These three-particle bound states can be considered as analogs of the hydrogen atomic $\mathrm{H}^{-}$and molecular $\mathrm{H}_{2}^{+}$ions, respectively. The application of a magnetic field $B$ changes the hydrogenic spectra drastically (see, e.g., Refs. [6 8]). As an example, whereas at $B=0$ the $H^{-}$supports in $3 \mathrm{D}$ only one bound singlet state, at any finite $B$ there appear [7] bound triplet $H^{-}$states and infinite number of quasi-bound states (resonances) associated with higher Landau levels (LL's). In 2D systems, it is the $X^{-}$triplet that becomes the ground state in high magnetic fields [2,3]. Singlet and triplet $X^{-}$and $X^{+}$states are observed in 2D systems by means of interband optical magneto-spectroscopy [1]. Intraband magneto-spectroscopy, in which internal transitions from populated (ground) to excited states are induced by a photon, can provide additional information about binding of hydrogen-like complexes. In the $\sigma^{+}$ polarization internal transitions in high $B$ are predominantly induced to the next electron LL. Such photoionizing bound-to-continuum $X^{-}$singlet and triplet transitions in the far-infrared (FIR) have been predicted theoretically and recently observed experimentally in GaAs quantum wells 任. In this work we describe some general implications of the existing exact symmetry - magnetic translations - for internal transitions of charged mobile $e-h$ complexes in $B$. We also present theory predictions for internal transitions from the $X^{-}$ground triplet state in the $\sigma^{-}$polarization in high $B$. A preliminary account of some of these results and implications for interband magneto-optics of $X^{-}$have been reported in Ref. [5].

Consider the Hamiltonian of interacting particles of charges $e_{i}$ in a magnetic field $\mathbf{B}$

$$
H=\sum_{i} \frac{\hat{\boldsymbol{\pi}}_{i}^{2}}{2 m_{i}}+\frac{1}{2} \sum_{i \neq j} U_{i j}\left(\mathbf{r}_{i}-\mathbf{r}_{j}\right),
$$

here $\hat{\boldsymbol{\pi}}_{i}=-i \hbar \boldsymbol{\nabla}_{i}-\frac{e_{i}}{c} \mathbf{A}\left(\mathbf{r}_{i}\right)$ and interparticle interactions potentials $U_{i j}$ can be rather arbitrary. In the symmetric gauge $\mathbf{A}=\frac{1}{2} \mathbf{B} \times \mathbf{r}$ the total angular momentum projection $M_{z}$, an eigenvalue of $\hat{L}_{z}=\sum_{i}\left(\mathbf{r}_{i} \times-i \hbar \boldsymbol{\nabla}_{i}\right)_{z}$, is an exact quantum number. In a uniform $\mathbf{B}=(0,0, B)$ the Hamiltonian (1) is also invariant under a group of magnetic translations whose generators are the components of the operator $\hat{\mathbf{K}}=\sum_{j} \hat{\mathbf{K}}_{j}, \hat{\mathbf{K}}_{j}=\hat{\boldsymbol{\pi}}_{j}-\frac{e_{j}}{c} \mathbf{r}_{j} \times \mathbf{B}$ (see, e.g., Refs. [6, /]). The operator $\hat{\mathbf{K}}$ is an exact integral of the motion, $[H, \hat{\mathbf{K}}]=0$, whose components commute in $B$ as

$$
\left[\hat{K}_{x}, \hat{K}_{y}\right]=-i \frac{\hbar B}{c} Q \quad, \quad Q \equiv \sum_{j} e_{j},
$$

while $\left[\hat{K}_{i p}, \hat{\pi}_{j q}\right]=0, p, q=x, y$. For neutral complexes (atoms, excitons, biexcitons) $Q=0$ and classification of states in $\mathbf{B}$ is due to the two-component continuous vector - the 2D magnetic momentum $\mathbf{K}=\left(K_{x}, K_{y}\right)$ [6, ]. For charged systems $Q \neq 0$ and the components of $\hat{\mathbf{K}}$ do not commute, which determines the macroscopic Landau degeneracy of exact eigenstates of (1). Using a dimensionless operator $\hat{\mathbf{k}}=\sqrt{c / \hbar B|Q|} \hat{\mathbf{K}}$ whose components are canonically conjugate, one obtains raising and lowering Bose ladder operators $\hat{k}_{ \pm}=\left(\hat{k}_{x} \pm i \hat{k}_{y}\right) / \sqrt{2}:\left[\hat{k}_{-}, \hat{k}_{+}\right]=$ $Q /|Q|$ (see (5) below). Therefore, $\hat{\mathbf{k}}^{2}=\hat{k}_{+} \hat{k}_{-}+\hat{k}_{-} \hat{k}_{+}$ has the oscillator eigenvalues $2 k+1, k=0,1, \ldots$. Since $\left[\hat{\mathbf{k}}^{2}, H\right]=0$ and $\left[\hat{\mathbf{k}}^{2}, \hat{L}_{z}\right]=0$, the exact charged eigenstates of (11) can be simultaneously labeled by the discrete quantum numbers $k$ and $M_{z}$ [7].

The usual optical selection rules for the dipole-allowed transitions in the Faraday geometry (the light propagates along B) are conservation of spin and $\Delta M_{z}= \pm 1$ for leftand right-circularly polarized light $\sigma^{ \pm}$. There is an additional selection rule: the quantum number $k$ is conserved. 
Indeed, the Hamiltonian $\hat{V}^{ \pm}=\sum_{i}\left(e_{i} \mathcal{F}_{0} \hat{\pi}_{i}^{ \pm} / m_{i} \omega\right) e^{-i \omega t}$ describing the interaction with the light of polarization $\sigma^{ \pm}\left(\mathcal{F}_{0}\right.$ is the radiation electric field, $\left.\hat{\pi}_{i}^{ \pm}=\hat{\pi}_{i x} \pm i \hat{\pi}_{i y}\right)$ commutes with $\hat{\mathbf{K}}_{i}$ and, therefore

$$
\left[\hat{V}^{ \pm}, \hat{\mathbf{k}}^{2}\right]=0 \Rightarrow k \text { is conserved } .
$$

In fact the perturbation $\hat{V}=F\left(\hat{\boldsymbol{\pi}}_{i}, t\right)$ can be an arbitrary function of the kinematic momentum operators $\hat{\boldsymbol{\pi}}_{i}$ and time $t$. In some limiting cases (e.g., at low fields $B$ ) $k$ can be associated [7] with the center of the cyclotron motion of a charged system as a whole. This gives some physical insight into its conservation. This selection rule is applicable to any charged $e-h$ system in $B$. In particular, it applies to mobile complexes - charged excitons $X^{-}$, $X^{+}$, multiply-charged excitons $X^{-n}$ (i.e., bound complexes $(n+1) e-h$ with $n>1$, which can exist in special quasi-2D geometries [9]), and to charged multipleexcitons $X_{N}^{-}\left(X_{N}-e\right)$, which exist in 2 D systems in high $B$ [2]. In deriving (3) we only used translational invariance in the plane perpendicular to $\mathbf{B}$. Therefore, relation (3) holds in arbitrary magnetic fields and for systems of different dimensionality, including semiconductors with a complex valence band described by the Luttinger Hamiltonian [10]. The selection rule (3) is also valid for internal transitions in electron systems. Note that for a translationally invariant one-component (e.g., electron) system with constant charge-to-mass ratio $e_{i} / m_{i}=$ const, the well-known Kohn theorem [11] states that internal transitions can occur only at the bare electron cyclotron $(e-$ CR) energy $\hbar \omega_{\mathrm{ce}}=\hbar e B / m_{e} c$. This is a consequence of the operator algebra $\left[H, \hat{V}^{ \pm}\right]= \pm \hbar \omega_{\mathrm{ce}} \hat{V}^{ \pm}$involving the center-of-mass inter-LL ladder operators. On the other hand, relation (3) is based on the algebra of intra-LL ladder operators. However, for one-component systems the center-of-mass decouples from internal degrees of freedom in $B$ and both theorems - though based on different operator algebras - give equivalent predictions in this case.

To make further discussion concrete, we shall consider transitions in the $\sigma^{-}$polarization in a 2D three-particle $2 e-h$ system with the Coulomb interactions, for a simple valence band, and in the limit of high magnetic fields:

$$
\hbar \omega_{\mathrm{ce}}, \hbar \omega_{\mathrm{ch}},\left|\hbar \omega_{\mathrm{ce}}-\hbar \omega_{\mathrm{ch}}\right| \gg E_{0}=\sqrt{\frac{\pi}{2}} \frac{e^{2}}{\epsilon l_{B}},
$$

where $l_{B}=\left(\frac{\hbar c}{e B}\right)^{1 / 2}$. Then mixing between different LL's can be neglected and the $X^{-}$states can be classified according to the total electron and hole LL numbers, $\left(N_{e} N_{h}\right)$. The corresponding basis for $X^{-}$is of the form [12] $\phi_{n_{1} m_{1}}^{(e)}(\mathbf{r}) \phi_{n_{2} m_{2}}^{(e)}(\mathbf{R}) \phi_{N_{h} M_{h}}^{(h)}\left(\mathbf{r}_{h}\right)$, and includes different three-particle $2 e-h$ states such that the total angular momentum projection $M_{z}=N_{e}-N_{h}-m_{1}-m_{2}+M_{h}$, and LL's $N_{e}=n_{1}+n_{2}, N_{h}$ are fixed. Here $\phi_{n m}^{(e, h)}$ are the $e$ - and $h$ - single-particle factored wave functions in $B ; n$ is the LL quantum number and $m$ is the oscillator quantum number $\left[m_{z e(h)}=\underset{(-)}{+}(n-m)\right]$. We use the electron relative and center-of-mass coordinates: $\mathbf{r}=\left(\mathbf{r}_{e 1}-\mathbf{r}_{e 2}\right) / \sqrt{2}$ and $\mathbf{R}=\left(\mathbf{r}_{e 1}+\mathbf{r}_{e 2}\right) / \sqrt{2}$. Permutational symmetry requires that for electrons in the spin-singlet $s$ (triplet $t$ ) state the relative motion angular momentum $n_{1}-m_{1}$ should be even (odd). To make this basis compatible with magnetic translations, i.e., to fix $k$, an additional Bogoliubov canonical transformation should be performed [5].

The calculated eigenspectra of the three-particle $2 e^{-}$ $h$ states with two electrons in the spin-triplet state $t$ $\left(S_{e}=1\right)$ are shown for two lowest $\left(N_{e} N_{h}\right)=(00),(01)$ LL's in Fig.1. The spectra consist of continua, which correspond to the motion of a neutral magnetoexciton (MX) [A] 8 . In addition, there are discrete bound $X^{-}$ states lying outside the continua in which the internal motions of all particles are finite. The continuum in the $\left(N_{e} N_{h}\right)=(00)$ LL consists of a MX band of width $E_{0}$ extending down in energy from the free (00) LL and corresponding to the $1 s \mathrm{MX}\left(N_{e}=N_{h}=0\right)$ [ 8 plus a scattered electron in the zero LL, labeled $X_{00}+e_{0}$. In the next hole LL $\left(N_{e} N_{h}\right)=(01)$, the MX band is of width $0.574 E_{0}$ [8] and corresponds to the $2 p^{-}$MX $\left(N_{e}=0\right.$, $N_{h}=1$ ) plus a scattered electron in $N_{e}=0 \mathrm{LL}$, labeled $X_{01}+e_{0}$. Moreover, there is a band above each free LL originating from the bound internal motion of two $2 \mathrm{D}$ electrons in $B$ (labeled $2 e+h_{N_{h}}$ ) 㺻. The spectra of the discrete bound $X^{-}$states are the following. In the lowest $\left(N_{e} N_{h}\right)=(00)$ LL there exists only one bound $X_{t 00}^{-}$triplet state, lying below the lower edge of the $1 s$ MX band, the binding energy of which is $0.043 E_{0}$ [2, 3. In the next hole LL $\left(N_{e} N_{h}\right)=(01)$ the $2 p^{-}$MX band is narrow, and there appear many bound $X_{t 01}^{-}$states lying below the continuum edge (Fig. 1). This should be contrasted with the situation in the next electron LL $\left(N_{e} N_{h}\right)=(10)$, where only one bound triplet state $X_{t 10}^{-}$exists 顿.

In the $\sigma^{-}$polarization, the $h-\mathrm{CR}$-like inter-LL $\Delta N_{h}=$ 1 transitions are strong and gain strength in $B$. These are allowed by the usual selection rules: spin conserved, $\Delta M_{z}=-1$. Consider first the photoionizing $X^{-}$transitions in which the final three-particle states belong to the $X_{01}+e_{0}$ continuum. There is an onset at the edge indicated in Fig. 1 by transition 3. It occurs at an energy $\hbar \omega_{\mathrm{ch}}+0.469 E_{0}$, i.e. above the $h-\mathrm{CR}$ at an energy that equals the difference in the $1 s$ and $2 p^{-}$MX binding energies, plus the $X_{t 00}^{-}$binding energy. This transition may be thought of as the $1 s \rightarrow 2 p^{-}$internal transition of the MX [13], which is shifted and broadened by the presence of the second electron. Photoionizing transitions to the $2 e+h_{1}$ band have extremely small oscillator strengths and can be neglected.

In order to understand bound-to-bound transitions $X_{t 00}^{-} \rightarrow X_{t 01}^{-}$, let us describe the structure of the $X^{-}$ states in more detail. Generally, there exist families of macroscopically degenerate $X^{-}$states in $B$. Each $i$-th family starts with its Parent State (PS) $\left|\Psi_{M_{z}}^{\left(P_{i}\right)}\right\rangle$, which 


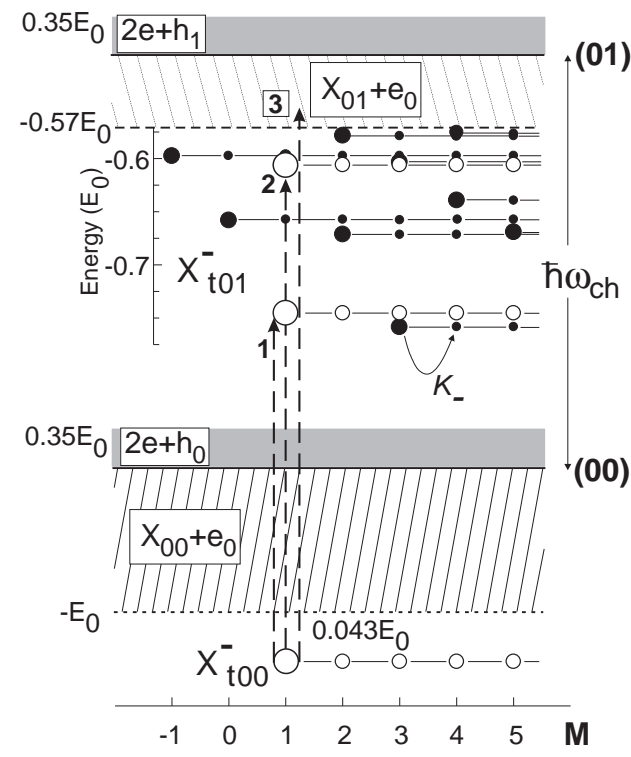

FIG. 1. Schematic drawing of bound and scattering electron triplet $2 e-h$ states in the lowest LL's $\left(N_{e} N_{h}\right)=(00),(01)$. The quantum number $M=-M_{z}$ for the $\left(N_{e} N_{h}\right)=(00)$ states and $M=-M_{z}-1$ for the $\left(N_{e} N_{h}\right)=(01)$ states. Large (small) dots correspond to the bound parent $k=0$ (daughter $k=1,2, \ldots) X^{-}$states. Allowed strong transitions in the $\sigma^{-}$ polarization must satisfy $\Delta N_{h}=1, \Delta M_{z}=-1$, and $\Delta k=0$. Filled dots in the (01) LL correspond to families of dark $X_{t 01}^{-}$ states (see text).

has $k=0$ and $M_{z}$ has its maximal possible value for that family (cf. with translationally invariant states in $2 \mathrm{D}$ electron systems in strong $B$ [14). The normalized daughter states with $k=1,2, \ldots$ in the $i$-th family, $\left|\Psi_{M_{z}^{\prime}}^{\left(D_{i}\right)}\right\rangle$, are constructed from the PS with the help of the ladder operators:

$$
\left|\Psi_{M_{z}-l}^{\left(D_{i}\right)}\right\rangle=\frac{1}{\sqrt{l !}} \hat{k}_{-}^{l}\left|\Psi_{M_{z}}^{\left(P_{i}\right)}\right\rangle,
$$

where we have used $\left[\hat{L}_{z}, \hat{k}_{-}\right]=-\hat{k}_{-}$. Conservation of $k$ implies therefore that internal transitions in the $\sigma^{ \pm}$ polarization, satisfying the usual selection rules $\Delta M_{z}=$ \pm 1 , spin conserved, are allowed only between states from families such that their PS's are connected by a dipole transition $\left|\Psi_{M_{z}}^{\left(P_{j}\right)}\right\rangle \rightarrow\left|\Psi_{M^{\prime}}^{\left(P_{i}\right)}\right\rangle$, i.e., have $M_{z}^{\prime}=M_{z} \pm 1$. Indeed, for the transition dipole matrix element between the daughter states in the $m$-th and $n$-th generations we have from (5):

$$
\begin{aligned}
\mathcal{D}_{i j}= & \left\langle\Psi_{M_{z}^{\prime}-m}^{\left(D_{i}\right)}\left|\hat{V}^{ \pm}\right| \Psi_{M_{z}-n}^{\left(D_{j}\right)}\right\rangle= \\
& \frac{1}{\sqrt{n ! m !}}\left\langle\Psi_{M_{z}^{\prime}}^{\left(P_{i}\right)}\left|\hat{k}_{+}^{m} \hat{V}^{ \pm} \hat{k}_{-}^{n}\right| \Psi_{M_{z}}^{\left(P_{j}\right)}\right\rangle .
\end{aligned}
$$

From the commutativity $\left[\hat{V}^{ \pm}, \hat{k}_{-}\right]=\left[\hat{V}^{ \pm}, \hat{k}_{+}\right]=0$ we see that either $\hat{k}_{-}$annihilates the left-PS $(n>m)$ or

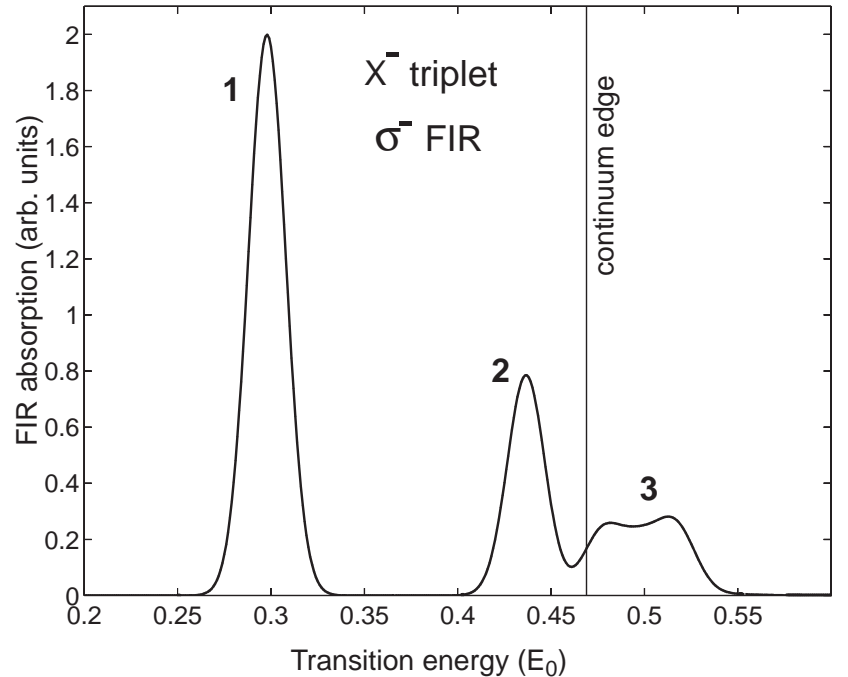

FIG. 2. Energies (counted from $\hbar \omega_{\mathrm{ch}}$, in units of $\left.E_{0}=\sqrt{\pi / 2} e^{2} / \epsilon l_{B}\right)$ and dipole matrix elements of the inter-LL $\Delta N_{h}=1$ transitions (Fig. 1) from the ground triplet $X_{t 00}^{-}$state in the high-field limit. The spectra have been convoluted with a Gaussian of the $0.02 E_{0}$ width.

$\hat{k}_{+}$annihilates the right-PS $(n<m)$ in (6). Therefore, $\mathcal{D}_{i j}=0$, unless $n=m$ and $M_{z}^{\prime}-M_{z}= \pm 1$. From the operator algebra it is clear that $\mathcal{D}_{i j}$ is the same in all generations and, thus it characterizes the two families of states.

This selection rule, due to the rich structure of the continuum, is easily satisfied for bound-to-continuum transitions. However, considering many families of the bound $X_{t 01}^{-}$states in the next hole LL $\left(N_{e} N_{h}\right)=(01)$, we see that there are only two PS's that are connected by the FIR transition (Figs. 1,22). Therefore all families except these two are dark, i.e., are not accessible by internal transitions from the ground $X_{t 00}^{-}$bound states: There exist only two strong bound-to-bound $X_{t 00}^{-} \rightarrow X_{t 01}^{-}$transitions in the $\sigma^{-}$polarization, both lying above the $h-\mathrm{CR}$ (cf. [1]). Breaking of translational invariance (e.g., by impurities) would make transitions to many dark states possible and lead to drastic changes in the absorption spectra. We note also that in $2 \mathrm{D}$ systems the $X^{-}$states belonging to higher LL's are genuinely discrete only in sufficiently strong $B$ [cf. (4)]. With decreasing $B$, the discrete states merge with the MX continuum of lower LL's and become resonances. Such a situation is also typical for bulk 3D systems, where $X^{-}$(and $H^{-}$) states in higher LL's always merge with the continuum of the unbound internal $z$-motion in lower LL's [7]. Since the quantum number $k$ is still rigorously conserved, the selection rule predicts which of the resonances are dark and which are not. The absorption spectra in such situation can have an asymmetric form typical for Fano-resonances. 
In conclusion, we have demonstrated that internal optical transitions of charged mobile complexes and ions in magnetic fields are governed by a novel exact selection rule, a manifestation of magnetic translational invariance. Internal bound-to-bound transitions of charged excitons $X^{-}$in 2 D systems in $B$ should be very sensitive to breaking of translational invariance (by impurities, disorder etc.). This can be used for studying the extent of $X^{-}$localization in quantum wells.

We thank S.M. Apenko, B.D. McCombe, D.M. Whittaker, and D.R. Yakovlev for helpful discussions. This work was supported by RBRF Grant \#97-2-17600 and the "Nanostructures" Grant \#97-1072. ABD is grateful to the Humboldt Foundation for research support.

[1] G. Finkelstein, H. Shtrikman, and I. Bar-Joseph, Phys. Rev. Lett. 74, 976 (1995) A. J. Shields, M. Pepper, M. Y. Simmons, and D. A. Ritchie, Phys. Rev. B 52, 7841 (1995); D. R. Yakovlev, V. P. Kochereshko, R. Suris et al. Phys. Rev. Lett. 79, 3974 (1997); O. V. Volkov, V. E. Zhitomirskii, I. V. Kukushkin et al. Pis'ma ZhETF, 67, 707 (1998) [JETP Lett. 67, 744 (1998)].

[2] J. J. Palacios, D. Yoshioka, and A. H. MacDonald, Phys. Rev. B 54, R2296 (1996).

[3] D. M. Whittaker and A. J. Shields, Phys. Rev. B 56, 15185 (1997).

[4] A. B. Dzyubenko, A. Yu. Sivachenko, H. A. Nickel, T. M. Yeo, G. Kioseoglou, B. D. McCombe, and A. Petrou, cond-mat/9907124; Proceedings EP2DS-13 (Ottawa, 1999), Physica E 6, 156 (2000).

[5] A. B. Dzyubenko and A. Yu. Sivachenko, condmat/9908406 Proceedings EP2DS-13, Physica E 6, 226 (2000).

[6] L. P. Gor'kov and I. E. Dzyaloshinskii, Zh. Eksp. Teor. Fiz. 53, 717 (1967) [Sov. Phys. JETP 26, 449 (1968)].

[7] J. E. Avron, I. W. Herbst, and B. Simon, Annals of Phys. 114, 431 (1978).

[8] I. V. Lerner and Yu. E. Lozovik, Zh. Eksp. Teor. Fiz. 78, 1167 (1980) [Sov. Phys. JETP 51, 588 (1980)].

[9] V. I. Yudson, Phys. Rev. Lett. 77, 1564 (1996).

[10] J. M. Luttinger, Phys. Rev. 102, 1030 (1956).

[11] W. Kohn, Phys. Rev. 123, 1242 (1961).

[12] A. B. Dzyubenko, Phys. Lett. A 165, 357 (1992); 173, 311 (1993).

[13] A. B. Dzyubenko, Pis'ma ZhETF 66, 588 (1997) [JETP Lett. 66, 617 (1997)].

[14] S. A. Trugman and S. Kivelson, Phys. Rev. B 31, 5280 (1985). 